\title{
Affluent gynaecological conditions
}

\author{
Dr Abri de Bruin \\ Reproductive \& Endocrine Unit, Department of Obstetrics \& Gynaecology, Faculty of Health Sciences, University of Pretoria, \\ Pretoria, South Africa
}

Diseases of affluence are those diseases which are thought to be a result of increasing wealth in a society, in contrast to diseases of poverty which result from impoverishment.

In 2005, the World Health Organisation (WHO) estimated that worldwide, 35 million people would die of chronic diseases such as diabetes, cancer and cardiovascular diseases, with $80 \%$ of the fatalities occurring in low- and medium-income countries (LMICs). It is known that our lifestyles and diets are changing. This is very true in the urban affluent group of people. This consequently raises the risk of chronic diseases.

South Africa shows an interesting disease pattern. Communicable diseases (can be passed from person to person) like AIDS and TB that is typical in a developing country, remains a major problem. However, we also have a heavy chronic disease burden as seen in a rich country.

What is an affluent condition? Is it a condition that only affluent people get? What is the reason that often in training hospitals we see so many different diseases than in private practice? Does this mean that we see "affluent diseases" in private practice and poor diseases in the state hospitals? Does this really mean that there are different diseases or do access to medical services and different modalities that are available in private practice lead to the fact that the same diseases are seen earlier in private practice? Will the social and work circle in which you operate lead to or predispose you to certain conditions? There may be reasons for diseases to be apparently affluent in nature while those may exist in the less affluent sectors of the population.

If one looks at the typical "affluent diseases", most of them are related to cardiovascular disease and lung conditions that are linked with lifestyle changes. However, there certainly are gynaecological conditions that fall in this category. Typically the gynaecological conditions do not form part of the most common diseases or causes of death and are thus neglected in many articles written on affluent diseases. Chronic diseases of lifestyle include heart disease, diabetes, cancer and stroke. These conditions tend to be more prevalent in populations that live longer. However, they are also much

\section{Correspondence}

Abri de Bruin

email: drabri@webmail.co.za more common among people that live affluent lifestyles. They tend to be less physically active and have a more unhealthy diet.

Traditionally, diseases of affluence have been thought to be more prevalent in developed countries but in the last ten years there has been a spike in numbers for virtually all in fast-growing developing countries such as China and India. In addition, in many developed countries the incidence of diseases of affluence like obesity, diabetes, and heart disease and some cancers are ironically higher among the poorest section of society. Food is abundant and cheap, but of such poor quality as to have minimal nutrition

The next relevant question is if there are only affluent diseases or are there affluent surgical procedures as well, that is not necessarily related to so-called affluent diseases?

\section{Medical Conditions Endometriosis}

Why is it so common in private practice while in training hospitals, not as many patients are seen with endometriosis? Contributing factors to this puzzle include less laparoscopies done in the public sector for pelvic pain and dysmenorrhea; this may decrease the frequency of diagnosis of this condition. In many instances, pelvic pain is diagnosed and treated as pelvic inflammatory disease without definite proof of the condition. There is not enough theatre time available for endoscopic surgery and a certain lack of equipment and trained staff in the training hospitals makes the ease of use of endoscopic equipment more difficult. So per se it may not just be an affluent condition, it is a very common condition, but the diagnosis and correct management is limited due to limited resources and training. In the infertility group of patients this is also true. Although we know that endometriosis is more prevalent in the infertile population than in non-infertile couples, these patients with infertility have more extensive workups done and the disease is diagnosed more often than in the non-infertile population. The use of the laparoscope as diagnostic tool has become common use. This leads to the condition being diagnosed more frequently and subsequently better managed.

\section{Infertility}

In affluent communities there is a trend for couples to start with a family later in life. First they decide to finish their 
studies and then when they start to work, they do not immediately start with a family. They first settle in their job and then they plan for pregnancies. This leads to a high number of couples with primary infertility older than 35years at first presentation. This is of course not true for everybody and this also does not mean that people that have children at an early age are not affluent. This is just a trend that is becoming more common to see. Especially with the increase in female people having more senior posts at work, not having children in this promotional time at work seems to be an important factor. Another important factor is the women who have more than one qualification or degree and the fact that they continue with their studies to a more advanced level. Studying and having a family is difficult, so many women tend to defer childbirth to later in life - after their studies.

Sometimes people will present with fertility concerns at a time when they feel comfortable with their qualifications and level at work. Now they see a fertility specialist after they have been trying for a year, but already they are in their late thirties.

Many women then have ovarian function issues and have to revert to donor oocytes or even surrogacy. The costs involved in these two modalities makes it impossible to afford for most couples. As fertility is not seen as a necessity by medical funders, people have to pay out of their savings for fertility treatment. So fertility treatment even without the expensive modalities of surrogacy and donor oocytes remains a condition that can only be afforded by affluent people. The sad part is that non affluent people will revert to borrowing money to pay for the chance to have a baby. In the end, this may lead to severe financial issues that can be devastating.

Family planning practices in the form of tubal ligations and vasectomies, coupled with the high incidence of divorce, lead to many patients that present with secondary infertility due to these factors. The fact that they can afford treatment in the form of reversal procedures makes this another interesting affluent gynaecological condition.

Another important aspect is the fact that money buys you steroids in the gymnasium or from the chemist. This is used more and more in training by young men with subsequent important negative impacst on their future fertility. It is a pity that image seems to be more important than being able to have a child. The only problem is this is not the thought process in a teenager in high school or a young man in his early twenties. It is also more and more common these days in middle aged men

\section{HPV and CIN-lesions}

Patients in private practice mostly attend a consultation annually for their cervical smears. Whether it can be assumed that they have insight in preventative health options and choose to be informed and pro-active or whether the benefits created by some funders assist in this process is unclear. Many of these patients are married or in a monogamous relationship and have a very small chance of developing cervical cancer or HPV. Consequently in the affluent sector with regular screening more CIN-lesions and less cervical cancers are detected. The pre-cancerous lesions can be treated correctly and there is less progression to cancer

The access to medical care and cervical smears and knowledge about the disease makes the detection of the condition more common in patients that have regular smears. Logistics also play a very important role. In private practice, a patient can make an appointment at a specific time to see a doctor. This can be accommodated in her work. In other words, you can make an appointment and see the doctor, get your examination and be back at work in a designated time. You do not have to go to the hospital, sit in a queue to register, sit in a queue to see the doctor, then sit in a queue to get medication and then you have spent a day at the hospital. This does not even take into account the difficulty the patient had to travel to the hospital.

Another important aspect is sexually transmitted diseases. While thought to be more common in indigent populations, affluence is changing that possible perspective. To what extend does access to drugs, clubs, social functions, clubs that offer sexual menus, add to the exposure of sex with multiple partners and thus increase the incidence of sexually transmitted diseases?

\section{PCOS}

The polycystic ovarian syndrome is certainly one of the diseases that are related to diet and lifestyle. Other factors play a major role including the genetic make-up of the individual. Again access to medical care makes the diagnosis more common in private patients and the management is earlier and lifestyle modifications are advised and implemented, not only from a fertility point, but also from a lifestyle point. Due to the fertility issues related to the disease, it also falls under the heading of infertility and the management in that context.

\section{Sexual dysfunction}

This is a group of conditions not frequently encountered in level two and three hospitals. To what extent this is related to affluence can definitely be seen in the context of private health and especially gynaecologists. Patients tend to develop a relationship with their gynaecologist that leads to trust. This will encourage patients to discuss these issues with less hesitancy. The management of this very complex issue is demanding and not often yielding satisfactory outcomes even in the affluent community.

\section{Fetal assessment and its sequels}

In private practice most pregnant women present to a gynaecologist between 6 and 10 weeks, much earlier than is common practice in the public sector. The time of first visit on its own has an important bearing on the management of the pregnancy itself including possibility of early detection of fetal abnormalities.

\section{Surgical Conditions \\ Cosmetic gynaecologic surgery}

Ahh, the elusive G-spot... A topic of discussion and research for years. Whether it actually exists is still open for debate. However, every week, women have medication injected in their "g-spot", supposedly to enhance sexual pleasure. At the same time, cosmetic surgery is performed to the labia and vagina. Does this enhance self-image, or is there a physical change or improvement? Due to the costs related to this and the fact that medical aids do not reimburse this, it has to fall in 
the category of an affluent surgical "disease" or rather condition, as the cost makes it unattainable for most women.

How big a role does the World Wide Web play? People are on blogs, hear what others say and many times false info gets patients to go for cosmetic vaginal procedures. Overseas specialists that have talk shows on TV also open the possibility of what is available and that is seen by many as the procedure to do.

\section{Laparoscopic surgery \\ Hysterectomy}

Most patients in permanent employment have access to private healthcare. However, due to work related issues, the luxury of 6 weeks of sick leave is not common. An international study that looked at return to work following hysterectomy found that the main factor influencing duration of sick leave was the qualification and job description of the patients. These patients tend to enquire about laparoscopic hysterectomy as they can return to normal activities much faster. In some cases women cannot undergo hysterectomy if they have to be off work for 6 weeks. There is of course no evidence that the common practice of "six weeks sick leave" as a recommendation is based on science.

\section{Oncologic surgery}

In an inverse situation of existence of cancer subspecialty units existing in the public sector private patients are often referred to those institutions for optimal care. However with the benefits of early detection following from regular followup some cases are now being considered for laparoscopic access surgery. So the fact that the more affluent people are screened earlier and managed at pre-cancerous stages, the patients with more advanced stages of cancer, can be helped by subspecialty units.

\section{Endometrial ablation}

Due to the ease of use as well as the fact that it is a day procedure with less complications than hysterectomy, a large number of endometrial ablations are done in private practice. Cost of the procedure as well as equipment issues make it more common in affluent patients that have access to private medical care.

\section{Tubal re-anastomosis}

This is requested more commonly in the affluent section of the population. Earlier tubal ligations are done due to completion of a family. However, the incidence of divorce is high and second and further marriages are common. In cases of female tubal ligations, tubal re-anastomosis is a relevant option and for male with vasectomies, re-anastomosis is also available. Again none of this is covered by medical aid and this has to be paid for personally by the patients. Affluence will give the opportunity for these procedures to be done. Of course the same requests and procedures are done in the public sector albeit less frequently.

\section{"Elective deliveries"}

\section{Elective caesarean section with its sequels}

Patients request caesarean sections. This is more and more common in private practice. Most of the time patients prefer it this way as it allows everything to be planned to fit their schedule. Whether it is due to work conditions or star signs or availability of anaethetists or the specific gynaecologist, if it is arranged, the couple feels comfortable with this arrangement. This is more expensive and arrangements like this increase the total spending on health care. It is typically not a consideration in the public sector hence the WHO concept that the caesarean section rate in a country should be low (below 20\%). Being affluent and having private healthcare makes this option a possibility despite the greater difficulties of costs, and in that sense a caesarean section on request can be seen as an affluent surgical procedure.

\section{Natural delivery units}

On the other hand there is a big drive towards the all-natural way of delivery. For this, patients pay huge amounts of money to deliver in a private setup where everything is managed by midwives. Sometimes this may include water births as well. Units like the private delivery units are not for free and having the money available makes this option available to certain patients.

\section{Final reflective thoughts}

As wealth increases, so does stress, and the risk for a host of Western lifestyle diseases. The best defence against these diseases is knowledge. To a certain extent for some of these conditions we should ask the question if it is really related to affluence or to access of medical care. There should be a greater effort to make access to facilities and experts in the field available to all women. The difference in wealth in our country is vast with the majority of people in South Africa not having access to private healthcare. With several disease factors associated with affluence these represent factors that a lot of people would rather not receive together with their affluence. Healthcare providers should continue to deliver optimal healthcare to all patients whatever the circumstances, with knowledge that these conditions will be predominantly seen in the private sector. 